\title{
Corrupción como proceso organizacional: comprendiendo la lógica de la desnormalización de la corrupción
}

\author{
Corruption as an organizational process: Understanding \\ the logic of the denormalization of corruption
}

David Arellano Gault

Centro de Investigación y Docencia Económicas, México

Recibido el 7 de abril de 2015; aceptado el 14 de enero de 2016

Disponible en Internet el 17 de septiembre de 2016

\begin{abstract}
Resumen
El artículo cuestiona los supuestos de la visión individualista de la corrupción y se introduce al contrario una conceptualización más «densa socialmente»: el proceso de normalización de la corrupción. En esta última lógica, se advierte que una definición de corrupción es siempre una definición política e ideológica que intenta imponer una visión determinada de comportamientos «adecuados» de acuerdo a una separación pretendidamente tajante y clara entre las esferas de lo público y lo privado. Se realiza una revisión de parte de la literatura sobre corrupción organizacional, con el fin de comprender cómo las rutinas, procesos y estructuras de socialización propias de la arena organizacional pueden llevar justamente a las personas a «caer en resbaladilla» hacia una dinámica de corrupción. A normalizarla en otras palabras. Se concluye con un llamado a discutir los procesos de desnormalización de la corrupción, como estrategia sustantiva más allá de la visión individualista o moralista del fenómeno.

Derechos Reservados (C) 2016 Universidad Nacional Autónoma de México, Facultad de Contaduría y Administración. Este es un artículo de acceso abierto distribuido bajo los términos de la Licencia Creative Commons CC BY-NC-ND 4.0.
\end{abstract}

Códigos JEL: M48; K42; Z13

Palabras clave: Corrupción; Corrupción organizacional; Socialización; Racionalización; Estrategias contra la corrupción

Correo electrónico: david.arellano@ cide.edu

La revisión por pares es responsabilidad de la Universidad Nacional Autónoma de México. 


\section{Abstract}

This article discusses the basic assumptions of an individualist vision on corruption. A different argument based on "social density" of the phenomenon is proposed instead: the process of normalization of corruption. Under this umbrella, corruption is a political concept that looks to impose a particular vision on what are "right" behaviors based on a sharp and unrealistic separation of the public and private sphere. A review of the organizational literature on corruption is developed, with the aim of understanding how organizational processes of socialization triggers behaviors that make corrupt acts to appear as "normal" under the organizational logic. Persons find themselves in a "slippery slope", generating agreements and social dynamics that are able to produce corrupt logics under the normal life of an organization. A plea for discussing the social processes needed to "un-normalize" corruption is defended a conceptualization that goes beyond an individualist and moralist vision of the phenomenon.

All Rights Reserved () 2016 Universidad Nacional Autónoma de México, Facultad de Contaduría y Administración. This is an open access item distributed under the Creative Commons CC License BY-NC-ND 4.0.

JEL classification: M48; K42; Z13

Keywords: Corruption; Organizational corruption; Socialization; Rationalization; Strategies against corruption

\section{Introducción}

Es común en la literatura sobre el tema de corrupción (por ejemplo, Nye, 1967; Rose-Ackerman, 1978) definirla como un acto de un individuo, un acto inapropiado o desviado de acuerdo a ciertos parámetros o valores sociales relativamente aceptados en una sociedad o agrupación. Esta definición incluso abre la posibilidad de comprender que, más allá de lo inapropiado del comportamiento del individuo en cuestión, puede considerarse bajo ciertas circunstancias como un comportamiento racional, calculado. En otras palabras, la corrupción desde este ángulo analítico es la decisión de un individuo en una transacción con otro individuo o individuos, aprovechándose (indebidamente se insiste) de una posición determinada en esa relación o transacción. Esta visión individualista se sustenta en un supuesto base de partida: que el individuo sabe o entiende en general la situación en la que está y ha calculado que comportarse corruptamente le conviene o le es benéfico. Se construye así un modelo de interacción muy particular entre actores que conscientemente calculan y actúan midiendo las consecuencias con cierta lógica e intención. De esta manera, al poner el énfasis en individuos calculadores y que actúan sustantivamente gracias a esa capacidad de cálculo (es decir, basado en un comportamiento consciente), la corrupción se ve como una decisión de individuos bajo un cierto contexto particular de valores. Ante esta lógica de individuos conscientes y calculadores se pueden inferir varias cosas: primero que la corrupción es inevitable en las relaciones sociales, pues está fundada en cálculos de actores racionales. Segundo, enfrentarla básicamente es un asunto de afectar los balances entre los costos y los beneficios que los individuos pueden calcular.

El punto central a considerar en este artículo está en que esta visión individualista es sumamente limitada, primero, para comprender el fenómeno en su integralidad y por lo mismo, como segundo punto, para proponer soluciones de largo plazo. El sentido principal del documento es mostrar, a través de una revisión de la literatura respecto de corrupción organizacional, que la corrupción no es un fenómeno de individuos entrando en relaciones discretas, sino un fenómeno social denso. Es una relación social, en otras palabras. Y esta lógica social es tan cierta para la corrupción en el 
sector privado como público, por lo que en este artículo no se hace una distinción particular sobre ello. En otras palabras, se habla de corrupción en organizaciones de cualquier tipo. A partir de la revisión de esta literatura organizacional de la corrupción, se busca justamente identificar cómo la corrupción puede normalizarse como un proceso social con etapas y procesos de socialización y racionalización.

La forma en que se desarrolla el argumento es a través de analizar cómo los estudios organizacionales de la corrupción ponen el énfasis más bien en el espacio grupal y social donde se da la decisión y donde se genera al final de cuentas el comportamiento. De esta manera, se llega a diagnósticos y conclusiones bastante diferentes y muchas veces contra intuitivas, respecto de la visión individualista. El punto de partida entonces está en que la corrupción se genera y florece en el mundo de las relaciones sociales, donde los individuos, su agencia, su comportamiento, están íntimamente vinculados y afectados por las interacciones y contextos donde se mueven y construyen su propia imagen, su propia voluntad (De Graaf, 2007). El comportamiento es un producto tanto racional, como emocional y relacional (Chugh et al., 2005, p. 79), donde las interacciones y las reglas y estructuras dentro de las cuales las personas se comportan importan. En este artículo, seguiremos la perspectiva de Chibnall y Saunders donde la corrupción es vista como una «clasificación negociada de un comportamiento antes que una cualidad inherente al comportamiento mismo» (Chibnall y Saunders, 1977, p. 139). En otras palabras, el concepto de corrupción es una construcción social muy particular de una sociedad que en su imaginario normativo ha intentado separar lo privado de lo público de una manera lo más clara y estricta posible. Solo así es viable encontrar definiciones que establecen que la corrupción, por ejemplo, es un abuso de una posición organizacional para ganar beneficios ilegítimamente (Anand et al., 2004, p.40). Esta definición clásica implícitamente habla de una clara diferenciación del beneficio privado sobre el colectivo o grupal, esencia permanente de las diferentes definiciones de corrupción contemporáneas ${ }^{1}$.

Discutir la enorme carga social y normativa que las definiciones clásicas de corrupción tienen, importa entonces. Decir que la corrupción es un acto indebido requiere definir «iindebido para quién, para qué, en qué contexto?». Lo que una visión basada en la idea de lo «indebido» implica entonces es que hay un parámetro externo, claro, legítimo, que envuelve al individuo y al grupo marcando con relativa claridad lo que convierte a un comportamiento como correcto o como corrupto. Si bien esto suena razonable y sencillo de comprender, en la práctica no lo es tanto. Por ejemplo, si es tan clara la definición de lo que es indebido debe ser claro definir el estatus contrario: ¿cómo se puede definir un estado de no-corrupción? (Philp, 1997). Dado que la respuesta a dicha pregunta no es sencilla se ha intentado como alternativa construir definiciones no del fenómeno de la corrupción como tal sino de algunas de sus características. Se ha intentado generar claras distinciones de lo que actos de legítimo beneficio significan, por ejemplo (utilizar estrategias políticas para subir puestos en la organización con tal de ganar más dinero y prestigio es legítimo, pero ayudar a un familiar a entrar en la organización a trabajar no). Una persona puede sospechar que está entrando en un conflicto de interés, por ejemplo, por una amistad que tuvo hace muchos años y que ahora, en una posición nueva, puede influir negativamente en su juicio. La negociación conceptual socialmente hablando implica para el individuo en esta situación evaluar

\footnotetext{
1 Andersson y Heywood (2009, p. 749) plantean que el intento de crear una definición global de corrupción de todas maneras se enfrentará a la variabilidad de las situaciones y tipos de corrupción: según si hablamos del sector público o privado, de un proceso administrativo o político; de los actores involucrados ya sean servidores públicos, empresarios, ciudadanos; de la gravedad y extensión de los actos (esporádicos, sistémicos, graves o simples violaciones). Añadiríamos a estas variaciones las causadas por las dinámicas organizacionales que aquí estudiamos.
} 
esas posibilidades al menos en 3 complejos pasos: primero, si la amistad puede influir; segundo, si esa influencia puede ser claramente ilegítima o solo probablemente impropia (a los ojos de ciertos actores); y tercero, definir cuáles pueden ser las consecuencias de dicha influencia. Cada paso implica una profunda reflexión y comprensión por parte del actor del contexto en el que se encuentra. Esto hace que sea factible proponer que toda definición de corrupción en el fondo lucha por negociar una clasificación de comportamientos, sospechando que esa clasificación algo explica de la naturaleza de los mismos.

De la misma manera, un contexto grupal u organizacional corrupto puede estar instalado antes de la llegada de un individuo a dicha organización. La relación social corrupta puede estar constituida de tal manera que sea normal (Bratsis, 2003, p. 17), que sea incluso una regla de la integración del grupo. En el caso de las organizaciones, la corrupción puede ser vista como tal por un grupo externo bajo ciertos parámetros, pero no serlo para aquellos que están dentro.

La persona está incrustada en espacios sociales como las organizaciones y en este sentido la lógica grupal impone una serie de posibilidades y condiciones a la manera en que las personas se manejan y presentan ante dichos espacios sociales. Existe por ejemplo, una importante literatura sobre la lógica antropológica, cultural y discursiva de la corrupción (De Graaf et al., 2010, p. 99; Andersson y Heywood, 2009; Haller y Shore, 2005; Sissener, 2001). La secrecía en una organización, por ejemplo, tiene que ver con las convenciones que regulan en ciertos grupos quiénes saben qué cosa, qué información poseen y porqué (Costas y Grey, 2014, p. 10). Las reglas y normas, formales e informales, del grupo o los grupos establecen esa «normalidad» de relaciones, que solo podrán ser vistas como «extrañas» a la luz de un código de referencia externo que establezca, por ejemplo, que esa secrecía puede ser una fuente de corrupción. La densidad social de la corrupción trae al frente del análisis argumentos incluso antiintuitivos tales como que la gente acusada de corrupción puede argüir que de hecho no sabía que estaba cometiendo un acto incorrecto.

El presente artículo tiene un método específico: realizar una revisión de la literatura organizacional sobre corrupción que es una literatura poco conocida en el ámbito del debate sobre el tema. Este método tiene la ventaja de introducir una serie de conceptos y discusiones que han sido poco debatidos en los espacios dominantes de debate sobre la corrupción, más tendientes a debates normativos e instrumentales. Este método escogido tiene sin embargo desventajas: la principal, la carencia de estudios empíricos específicos. Hemos desarrollado algunos de esos estudios en otro lugar (Arellano, 2012). Pero sin duda esperamos que este artículo genere incentivos para realizar más estudios empíricos, sobre todo para el caso de la corrupción en México y en América Latina.

Dicho esto, a partir de la revisión de la literatura sobre la corrupción organizacional, el artículo establece que es necesario, si se quiere enfrentar la corrupción en una organización o grupo de organizaciones, relativizar las estrategias que lo ven como un problema de individuos calculando costos y beneficios. Vista como un fenómeno social denso, un producto de relaciones sociales, la corrupción es un punto de llegada, no de partida. Para un actor en esta lógica, clasificar y comprender un acto determinado, y ser capaz de diferenciarlo como un acto honesto o deshonesto, implica que sea capaz de contrastarlo con los acuerdos y prácticas establecidos grupalmente como normales, en el día a día. Desnormalizar la corrupción parece ser entonces el centro de una estrategia que integralmente comprenda esta construcción colectiva del fenómeno y de los actos que lo componen.

El artículo se desenvuelve a través de 5 secciones incluida esta introducción. En la segunda sección se discute la organización como un proceso social de construcción de comportamientos en un entramado de reglas, sentidos y normas que genera la «normalidad» organizacional. Esta normalidad construida es el espacio donde, en la búsqueda de dar sentido al comportamiento, las 
organizaciones crean procesos sociales de interacción que intentan socializar a las personas en sus dinámicas, incluidas aquellas que pueden ser consideradas corruptas (tercera sección 3). En efecto, puede parecer antiintuitivo pero los procesos de socialización también funcionan para eventos como la corrupción, donde las personas racionalizan sus actos a través de darles sentido dentro de una trama organizacional. Esta racionalización permite a las personas reducir su disonancia cognitiva, es decir, el sentimiento de angustia ante la probabilidad de estar cometiendo un acto indebido (a partir de ciertos patrones internos o externos a la organización). En la cuarta sección, se explica cómo estos procesos de racionalización y socialización pueden entonces constituir una dinámica estable en el tiempo, una que va construyendo cierta trama de relaciones que se refuerzan unas a otras, constituyendo entonces una especie de «resbaladilla» donde cada vez más gente y de manera más frecuente cae o es atrapada en redes de actos corruptos. No solo se racionalizan y entran en la trama organizacional como normales sino que se hacen generalizados. $\mathrm{Y}$ en ese momento es probable hablar de una organización que ha hecho de la corrupción un acto normal y justificado (ha normalizado actos que vistos desde otros contextos o por otros actores son clasificables como actos corruptos). En la última sección se presentan algunas conclusiones respecto del impacto que el argumento de normalización de la corrupción puede tener en las estrategias para enfrentar este fenómeno que socialmente despierta cada vez más atención y preocupación.

\section{¿Organizaciones con individuos corruptos u organizaciones corruptas?}

Las razones por las que un individuo realiza acciones deshonestas son múltiples y diversas. La corrupción, en efecto, es un fenómeno social: un individuo decide ser corrupto o realiza una acción deshonesta (veremos que hay una diferencia entre ambas) siempre en un contexto donde sus reacciones sicológicas, sus experiencias, sus valores y las interrelaciones sociales que vive y sufre constantemente, forman parte de la ecuación (Rest, 1986).

Berger y Luckman (1978) son claros al respecto de cómo toda interacción humana inicia, es y se define dentro de una lógica social: las interacciones generan lazos entre las personas. Lazos que duran un segundo o décadas. Lazos capaces de crear eventualmente grupos y sub grupos que comparten objetivos o creencias. Estos lazos pueden ser vistos también como dinámicas de socialización, es decir, de autoorganización (Goffman, 1983): los lazos una vez creados tienden a estabilizarse y pueden reproducirse hacia otras personas conforme se van comunicando y compartiendo, por ejemplo, a través de rutinas. Aquí la palabra socialización implica la construcción y reconstrucción de percepciones y eventualmente comportamientos que los individuos al crear lazos inducen, entienden y generan con el fin de intentar llevar la relación entre ellos a alguna (o algunas) arena particular, de acuerdo a la perspectiva de cada agente involucrado.

El proceso de socialización, al ser en el fondo sustantivamente situacional y contingente (depende de las personas concretas en las situaciones específicas en que se hallan), contiene elementos estabilizadores pero que en última instancia son cambiantes y modificables. De ahí que los mecanismos de socialización pueden ser contradictorios, opacos, antiintuitivos. Agencia y estructura (Giddens, 1984, p. 5) se interpenetran por lo que no hay garantía de que los procesos de socialización escapen a la propia dinámica de interacción. En otras palabras, los procesos de socialización pueden generar muy diversas reacciones de los diferentes agentes y crear efectos no esperados e incluso antiintuitivos para los propios actores involucrados. Esto es lo que suele suceder en las organizaciones que caen en lógicas de corrupción: han socializado prácticas y comportamientos que inducen a la corrupción, muchas veces como una consecuencia no esperada ni deseada de la acción. 
Siguiendo esta línea de reflexión, puede comprenderse cómo en un determinado marco organizacional de socialización, la definición de lo que es un comportamiento deshonesto está atada a una clasificación y estructuración dentro de lo que es organizacionalmente definido: un comportamiento es clasificado por algunos en ciertas circunstancias como tal porque viola ciertas normas morales organizacionalmente (o grupalmente) aceptadas de manera generalizada (todo esto no significa necesariamente que ese acuerdo sea unánime ni comprendido de la misma manera por todos; Kish-Gephart et al., 2010, p. 2) ${ }^{2}$. Los casos a discusión se multiplican entonces: personas que defienden que no sabían que los actos cometidos eran corruptos, actos que en un contexto particular son definidos como corruptos y en otro no, actos que temporalmente son vistos como aceptables pero que pasadas ciertas circunstancias lo dejan de ser.

Cómo se construye organizacionalmente sentido y socialización es importante por tanto de estudiar y comprender, sobre todo si hablamos de normalización de actos como la corrupción. Se puede comenzar estableciendo que las organizaciones pueden ser vistas como criaturas sociales que se componen de gente comportándose (Simon et al., 2010, p. 55). Comportándose a partir de la combinación de las cargas y condiciones individuales de cada participante y de los lazos y relaciones que componen con otros, en un marco de cierta formalidad y determinado grado de dominación que busca, pretende, unir esas relaciones con cierto sentido (o sentidos) y en un marco de encuadramiento de lo «normal» y de lo «aceptable. En todo caso, en el marco organizado, las personas socializan sus pensamientos e intenciones dentro de un marco no rígido pero sí que pretende encuadrar los comportamientos en cierta dirección y con determinadas premisas. Es por ello que las personas componen a las organizaciones, pero las organizaciones son más que las personas que las componen: se convierten en una serie de lazos entre las personas, lazos sociales, grupales, individuales, contingentes pero pretendidamente estables en el tiempo.

De esta manera, cuando se habla de corrupción es prácticamente ineludible hablar de organización. Corruptor y corrompido, corruptos y víctimas, se encuentran tarde o temprano en el marco de organizaciones que los regula, vigila, alienta o intenta controlar. Comprender cómo se genera entonces la corrupción es intentar comprender la dinámica de los lazos y relaciones (temporales o semipermanentes) que se construyen entre los diferentes agentes para que dicha práctica sea posible de realizar e incluso posible de estabilizar (como la idea de corrupción sistémica insinúa).

$\mathrm{Al}$ introducir la variable organizacional los elementos sicológicos y grupales de la corrupción aparecen con claridad y sin tonos moralistas. Esto en la tradición de Goffman, quien trató de «leer» los procesos de presentación entre las personas como actos lógicos, incluyendo aquí el secreto, la maquinación y el acomodamiento de la fachada que se utiliza para presentarse en una reunión grupal dentro de la organización. Por ejemplo, el proceso de ocultamiento y engaño (deceit) que suele llevar a las organizaciones a sistematizar actos de corrupción no se genera en un solo acto sino que va avanzando de una mentira no detectada, a mentiras mayores para soportar la anterior mentira (Messick y Bazerman, 1996, p. 21). Difícilmente una mentira o un engaño termina siendo una actividad individual en una organización: conforme el engaño va involucrando a más

\footnotetext{
${ }^{2}$ La densidad del fenómeno de la corrupción, visto desde el ángulo organizacional, no hace sino complejizar todavía más su comprensión. Por ejemplo, sería necesario diferenciar acto corrupto de otros muy similares como comportamiento inapropiado (por ejemplo, el acoso laboral), comportamiento organizacional desviado (simular que se trabaja, por ejemplo) o comportamiento antisocial (e.g. bullying) (Ackroyd y Thompson, 1999). Las fronteras no son rígidas y, por supuesto, como procesos organizacionales, los 3 ejemplos anteriores pueden derivar en un acto corrupto. Algunos autores (Pinto, Leana y Pil, 2008, p. 687) lo diferencian debido a la obtención de una ganancia financiera. Esto implicaría que corrupción organizacional de individuos podría ir desde sobornos, maquillaje financiero, sobreestimación de gastos u horas trabajadas, fraude e incluso el robo. Corrupción organizacional en beneficio de la organización serían el fraude corporativo, el crimen de negocios y la desviación del negocio hacia actividades criminales.
} 
personas, compañeros e incluso autoridades pueden comenzar a ejercer presión para mantener la red de mentiras (Cialdini et al., 2004, pp. 70-71). El lenguaje puede ir cambiando, los agentes organizacionales cambian las palabras para hacerlas parecer no como mentiras o cuestionables sino más «higiénicas» (por ejemplo, en vez de hablar de soborno hablar de «engrasar la máquina», Bandura, 1990). Estos elementos al final de cuentas van mostrando cómo las mentiras y el engaño pueden comenzar en una dimensión reducida, ir creciendo y luego irse enredando en la telaraña de relaciones personales y jerárquicas, cayendo poco a poco, como en resbaladilla, en dinámicas sociales e institucionalizadas de corrupción.

Dos metáforas al menos han tratado de simplificar estas explicaciones organizacionales (desafortunadamente algunas veces con un tono claramente moralista): el fenómeno de «la manzana podrida» o el fenómeno del «barril podrido» (bad apple y bad barrel). Una persona o personas que han decidido ser corruptas y violar las reglas organizacionales éticamente «adecuadas» sería la manzana podrida. En el caso del barril podrido, de lo que hablamos es de una organización (o alguna de sus estructuras o partes) que ha caído ya en una dinámica corrupta que se puede esconder incluso bajo un aura de acción ética o racional. De otra manera expuesto, corrupción contra la organización o corrupción a nombre de la organización (Pinto et al., 2008, p. 685). La cuestión en todo caso pareciera ser, más allá de estas etiquetas, comprender cómo una persona llega a ser una manzana podrida, algunas veces sin enterarse, y cómo en este proceso, el barril y su dinámica puede ser de alta importancia para tal efecto. En la siguiente sección nos introduciremos al proceso organizacional de la corrupción como se ha dibujado en los anteriores párrafos.

\section{Los procesos organizacionales de la corrupción: normalizando a través de racionalización y socialización}

La característica organizacional más antiintuitiva de la corrupción en organizaciones es que los actos de corrupción se hacen normales. La literatura a este respecto está llena de evidencias y referencias a que, por lo general, la gente que ha sido procesada por corrupción generalmente niega que haya realizado algún acto ilegal o incluso inmoral (Benson, 1985; Conklin, 1977; Cressey, 1986; Geis y Meier, 1979; Sykes y Matza, 1957).

Una de las explicaciones posibles para esta aparente paradoja puede encontrarse justamente en la capacidad de las organizaciones para rutinizar y normalizar las diferentes actividades, valores y objetivos de los individuos y los grupos que las integran. Así como una organización normaliza el respeto por la jerarquía o los procedimientos aceptables en el trabajo diario, esas mismas dinámicas de influencia parecen funcionar para convertir comportamientos clasificables como corruptos en comportamientos «normales». Más todavía, un grupo de comportamientos no corruptos puede estar combinándose con otros que sí lo son, y un individuo en particular puede no tener la fotografía completa de la interacción entre diversos agentes. Las personas en las organizaciones requieren construir el sentido de lo que hacen y su perspectiva es siempre limitada (por tiempo, recursos, capacidad, como dice el concepto clásico de racionalidad limitada de Simon, 1947). Así, una organización construye una serie de mecanismos de influencia, rutinas y principios que ayudan a los miembros de la organización a dar sentido a sus acciones, ahorrando tiempos a través de rutinas, propiciando la cooperación a través de procesos estandarizados, especializados y compartimentalizados. Todos estos, entre muchos otros mecanismos o instrumentos de influencia. Son estos mismos mecanismos los que, intencionalmente o no, pueden construir esquemas de normalización de redes de acción y comportamientos corruptos.

Desde este punto de partida, la acción organizacional es una construcción social a la Berger y Luckman (1978): los actores organizacionales construyen diferentes interpretaciones y lealtades 
para internalizar diferentes lógicas de socialización. El grupo, el subgrupo, el departamento, la organización como un todo, la industria donde la organización se mueve, y por último el país, la sociedad, son diferentes niveles donde el actor internaliza y socializa las normas, las reglas, las expectativas, los principios morales incluso, que le darán cabida en dicha colectividad (Chibnall y Saunders, 1977, p: 141). Evidentemente, esa socialización secundaria como lo llaman Berger y Luckmann implica un proceso que puede ser contradictorio de interpenetración en diferentes niveles de agregación. En corto, las lógicas de socialización de uno de los estratos (por ejemplo, la organización) pueden chocar con otro estrato (por ejemplo, el grupo). Acusar a un colega de corrupción, por ejemplo, puede ser lo adecuado para la organización como un todo, pero moralmente puede ser visto por el grupo como un acto de deslealtad. Maquillar algunas cifras financieras puede ser benéfico para la organización, bajo el argumento de que buena gente se equivocó con buenas intenciones, por ejemplo, pero visto como un acto de corrupción a nivel de la sociedad. ¿Cómo lidian los seres humanos con estas contradicciones fundamentales? Una posible respuesta es justamente la racionalización.

La racionalización es un acto de interpretación fundamental e indispensable para que un actor pueda ser un actor social. Al menos desde Goffman (1981) se comprende bien que los individuos saben presentarse ante los demás con una estrategia fundamental de claroscuros que permitan construir una imagen determinada y dar cierta certidumbre a la interacción esperada con los demás. ¿Por qué se obedece a un jefe? Porque se cree en la legitimidad de su jerarquía, a la Weber. Es una racionalización necesaria para la constitución del tejido de interacción social. Esa racionalización no deja de ser justamente una interpretación, una estrategia de sentido, una amalgama de esencia y apariencia de los que los actores desean se observe y desean permanezca oculto (la opacidad es un ingrediente fundamental de la interacción, Arellano, 2010; Costas y Grey, 2014). No solo la opacidad, sino el secreto y la secrecía forman parte inevitable e indispensable del repertorio de estrategias de interacción y comunicación en las organizaciones (Zerubavel, 2006). Cuando se habla de corrupción, por ejemplo, los actores en un grupo dentro de la organización pueden haber creado una racionalización que permite construir ese acto no como un acto corrupto sino como un accidente o un acto necesario para lograr un bien común mayor, con el fin de mantener cierto nivel de salud moral colectiva que justifique al grupo (Ashforth y Anand, 2003, p. 16).

El abanico de opciones de racionalización organizacional en casos de corrupción es diverso y amplio. Hagamos un resumen de lo que la literatura sobre el tema ha encontrado en diversas experiencias y casos (Anand et al., 2004, Ashforth y Anand, 2003; Ashforth et al., 2008; Felps et al., 2006, Fleming y Zyglidopoulos, 2009, Kish-Gephart et al., 2010; Pinto et al., 2008; Zyglidopoulos y Fleming, 2008, y Zyglidopoulos et al., 2009).

\section{Negación de la responsabilidad}

Este es el clásico desplazamiento que puede hacer una persona para establecer que era parte de una gran maquinaria donde no se tenía la posibilidad de entender que se estaba realizando un fraude o un acto de corrupción. $\mathrm{O}$ argüir que en realidad estaba siguiendo órdenes o que se estaba realizando lo que muchos otros estaban haciendo y por tanto que era un acto normal. En todo caso, se racionaliza que no se tenía control sobre la situación. Un policía de tránsito que es de inmediato introducido por sus superiores y colegas a las reglas respecto de cuotas de sobornos a cumplir cada semana, puede tener ingredientes de este tipo de racionalización. 


\section{Negación del daño}

Los agentes que se ven en estas circunstancias pueden argüir que en realidad el acto del que se les está acusando es de tan poca monta, comparado por ejemplo con las ganancias o beneficios de la organización, que no es razonable decir que fue un robo o un acto corrupto. El «robo hormiga» o la solicitud en ventanilla gubernamental de un pago «para los refrescos» puede ser un ejemplo.

\section{Negación de víctimas}

Una versión clásica de esta racionalización es plantear que el receptor del acto ilegal o deshonesto es más deshonesto todavía, haciéndolo merecedor de dicho trato. Es decir, se afectó a alguien pero ese alguien no es una víctima, sino una instancia tan corrupta o tan grande y poderosa e injusta, que el acto clasificado de corrupción en realidad fue un acto casi de reivindicación ante las injusticias. El robo de recursos desde un sindicato a una gran organización que se niega a pagar mejores salarios a sus trabajadores puede ser un ejemplo de esta racionalización.

\section{Compensación social}

Esta racionalización es fuerte socialmente hablando, pero común: implica dar un peso o un valor relativo a las creencias o necesidades de otros. Si hay actores corruptos y poderosos, y las leyes se consideran injustas, entonces se racionaliza que puede estar justificado actuar de una manera aparentemente deshonesta, pues en realidad «los otros» son los verdaderamente corruptos. O cuando se justifica que un acto corrupto en realidad reduce mayores prejuicios a personas débiles (este pequeño soborno le evita a una persona relativamente indefensa peores consecuencias). Muchos de los «pequeños» sobornos pueden seguir esta lógica: con el soborno se «ayuda» al menos a que otros paguen menos y evitar incluso que el pago llegue a los «verdaderamente grandes corruptos».

\section{Lealtades más elevadas}

Los diferentes estratos de socialización que los individuos viven socialmente llevan a contradicciones entre lealtades a diferentes grupos. El espíritu de cuerpo puede llegar a ser más fuerte que lealtades a lógicas «mayores» como la ley o las normas. Es una muy contradictoria racionalización pero muy real: la ley se corrompe en el nombre de la justicia incluso, haciendo que los fines justifiquen los medios. Un policía puede negarse a acusar a otro por ese espíritu de cuerpo, o utilizar influencias deshonestas para forzar un veredicto ante el convencimiento de la culpabilidad de un acusado.

\section{La metáfora de la balanza}

Esta racionalización implica el cálculo que se puede realizar de las «aportaciones» de una persona que las considera tan grandes que «merece» una retribución particular (que a veces esa persona se ha tomado de manera ilegal). En otras palabras, un burócrata puede argüir que trabaja muchas horas, muchas más que los demás, por lo que el robo hormiga o el soborno que solicitaba estaba justificado como una compensación ante el esfuerzo extra realizado.

Evidentemente estas racionalizaciones pueden ser interpretadas como simples mentiras o justificaciones hipócritas. Sin embargo, la historia no es tan simple. Muchas veces, estas 
racionalizaciones son verdaderos mecanismos de interpretación y justificación, que permiten al actor sentirse mejor consigo mismo, reduciendo la angustia o disonancia entre sus valores generales y sus actos particulares (Gigerenzer, 2002). La negación en sicología, no es un acto exclusivamente de hipocresía sino en realidad un proceso mental y social de justificación y modificación de los parámetros de interpretación por parte de los seres humanos (Lerner y Tetlock, 1999, p. 263; Bargh y Chartrand, 1999, p. 475). El autoengaño, con todo lo antiintuitivo que suena, es una realidad fundamental estudiada ampliamente en la sicología como un acto de modificación de los parámetros mentales para evaluar la realidad (Eagleman, 2012, Lehrer, 2009, Ainslie, 2001). En otras palabras, las personas construyen historias y percepciones de tal manera que sean capaces de reducir e incluso eliminar (al menos temporalmente) la disonancia cognitiva o angustia que les produce saber que están cometiendo un acto indebido (Festinger, 1957). Dicha disonancia es una tensión interna de las ideas o emociones de la persona con respecto a lo que observa que es o puede hacer de la realidad. Al reducir la disonancia, es posible sentirse menos afectado moralmente, por ejemplo, ante un acto realizado en circunstancias particulares y en contextos específicos. Una persona, entonces, puede reducir la disonancia cognitiva si racionaliza que su acto de corrupción no fue tan grave, fue seguido por otros, ordenados por otros, o si forma parte de un acto de «justicia» redistributiva contra los «verdaderos malos». El punto central es que racionalización implica un esfuerzo real, creativo y con impactos efectivos en la interpretación de la persona respecto de la honestidad o no de un acto. Y esta racionalización puede ser reforzada ampliamente por la organización y sus grupos, de forma sistemática o implícita en las rutinas o prácticas.

\section{Haciendo a la organización corrupta: socialización o la resbaladilla de la corrupción}

El proceso mental y relacional que va haciendo a un acto corrupto no ser visto o racionalizado como tal por las personas puede ser reforzado ampliamente por la dinámica organizacional. Las organizaciones usualmente son vistas como instrumentos o máquinas semirrobóticas donde las personas son engrane de una maquinaria aceitada. Pero en realidad, las organizaciones son construcciones sociales que se asumen formalizadas pero que construyen intrincadas e intensas relaciones humanas y sociales para consensuar, formar grupos, internalizar comportamientos y valores, así como para convencer y dirigir al logro de objetivos a muchas personas que además tienen que saber interpretar y darle sentido a dichos objetivos (Weick, 2001).

Una persona que ingresa a una organización inicia un denso proceso de socialización. ¿Qué es la organización, quiénes las componen, qué grupos las manejan, cuáles son los valores imperantes de jerarquía, obediencia, cooperación, conflicto y negociación?, entre muchos otros procesos que cualquier persona se ve obligada a decodificar al entrar a una organización (Kunda, 1992). Estas fuerzas y dinámicas sociales que permiten a las personas ser introducidas y aprender y construir su rol y su papel dentro de la organización, funcionan para cuestiones formalizadas (como identificar la jerarquía y las reglas de comunicación), como también para la socialización de actos o comportamientos que puedan en el tiempo alimentar la corrupción. Esta contradicción es una de las más importantes para comprender, dado que explica bastante sólidamente por qué las personas en general acusadas de corrupción no se asumen como culpables de dichos actos. En otras palabras, no se habla solamente de cinismo o de cálculo de las personas para negar la corrupción. Tampoco solo del proceso sicológico que permite la racionalización como se analizó en la sección anterior. Estamos hablando además de los propios procesos organizacionales que permiten a una persona ser parte del grupo, de la organización, que actúan y funcionan para ubicar y posicionar al actor en tramas de relaciones y actores que al final de cuentas puedan ser clasificados 
como corruptos. Pero en este caso, actos corruptos que los propios procesos organizacionales, paradójicamente, ayudaron a cimentar, consolidar y hacerlos consuetudinarios.

En este apartado revisaremos 4 procesos clásicos de socialización de la corrupción (Milgram, 2005[1974]; Anand et al., 2004): agentificación, cooptación, incrementalismo y compromiso. Estos 4 procesos permiten en principio comprender cómo la dinámica social de introducción de una persona a la lógica organizacional se va dando poco a poco, como un verdadero proceso de aprendizaje de las reglas y las normas de interacción en un colectivo. La metáfora preferida es la de la «resbaladilla»: cómo los procesos mentales y sociales de racionalización pasan a ser reconstruidos y fortalecidos por la lógica organizacional de interacción, poco a poco. En el caso de la corrupción, las organizaciones pueden crear condiciones para que las personas «resbalen» paulatinamente a cometer actos corruptos en un proceso que facilita que dichos actos sean racionalizados y justificados como actos «normales» o al menos aceptables desde la lógica del grupo o incluso de la organización. Para que este efecto «resbaladilla» se genere es necesario que la persona se incorpore a las reglas y tradiciones o prácticas organizacionales, aceptándose como un agente incrustado o embebido como tal en dichas reglas y prácticas (más adelante se discutirá esto como agentificación). Gracias a este proceso, la persona en la organización sigue siendo aquel individuo que entró a ella, pero a la vez es otro: el que obedece, es aceptado, «sabe» interpretar y comprender la «cultura» o la «naturaleza» de la organización como un submundo donde muchos de sus comportamientos y sentimientos solo adquieren lógica mientras está dentro de la organización ${ }^{3}$.

\section{Agentificación organizacional}

La agentificación es el proceso sicológico y social que lleva a la persona a internalizar su rol dentro de la organización y a observar a esta y sus miembros con la legitimidad y la autoridad para dirigir su comportamiento. De alguna manera, es una de las claves organizacionales para construir los otros 3 procesos de socialización (cooptación, incrementalismo y compromiso). Veamos.

Las organizaciones pueden ser vistas como construcciones sociales que intentan formalizar diversos mecanismos de interacción humana con miras a obtener determinados fines relativamente especificados (pero nunca cerrados). Los mecanismos de interacción humana se constituyen al menos parcialmente en un juego intersubjetivo de expectativas mutuas entre los agentes: alguien busca algo que en cierta manera depende de la reacción del otro, por lo que intuye que se requiere y se depende en cierta manera de la reacción del otro. Esta es la base de interacción que genera lazos sociales, invisibles pero no por ello menos reales, que vinculan a los agentes. Esos lazos de la relación humana se juegan en diversos espacios sociales, muchos de ellos en relaciones cara a cara (Goffman, 1983). Son lazos que obligan a que la interacción muchas veces tenga que ser ilocucionaria o dramatizada, dependiente de sus propios juegos de máscaras, secretos y mentiras (que se han estudiado en la sociología sólidamente desde al menos del texto clásico de Simmel, 1906). Las organizaciones, así vistas, constituyen espacios que se pretenden formalizados, por lo que todos estos entrejuegos existen y se mantienen, pero en una faceta que basa su legitimidad en la formalización de las relaciones. El peso de lo formal no está, por tanto, en que desplaza o elimina la lógica «informal» o humana cara a cara o ilocucionaria: lo que hace la organización en todo caso es darle legitimidad a la faceta formal como ordenadora o piso estricto sobre el que el juego

\footnotetext{
${ }^{3}$ Este argumento que parece antiintuitivo ha sido también propuesto y estudiado en cuestiones como actos malvados o francamente inhumanos. Estudios sobre los médicos nazis (Lifton, 1986), la matanza de My Lai (Turse, 2013), entre otros (Staub, 1989) han argumentado que lo más desconcertante es encontrar no entes malvados intrínsecamente que desarrollan actividades terribles, sino justamente lo contrario: gente común atrapadas en fuerzas sociales y organizacionales.
} 
informal se juega. Una organización que es vista como legítima por las personas es una poderosa fuente de obediencia y por tanto de dirección de comportamientos hacia ciertas direcciones. El famoso experimento de Milgram, 2005[1974]) confirma cómo el peso de la autoridad dispara toda una serie de reacciones, pensamientos, supuestos y justificaciones que permiten a las personas racionalizar sus acciones a la luz de las personas y su autoridad y las reglas o normas que se defienden en su nombre. El «estado agentificado» (agentic state, Milgram, 2005, p. 133) es aquel donde el individuo, que puede considerarse a sí mismo como autónomo en ciertas circunstancias, una vez dentro de la organización, transforma su comportamiento y su racional a la lógica de la orden de la organización. Se pone, dice Milgram, en un estatus que permite la obediencia de la persona, algo que las organizaciones hacen muy bien.

La persona organizacional agentificada entonces es esa persona que «sabe» cómo comportarse en la organización. Y para ello, para «saber» y ser aceptado, requiere comportarse de ciertas maneras que muchas de las veces solo son comportamientos que aparecen mientras se está en la organización. No se trata acá de plantear una dicotomía, como a veces Milgram ha sido criticado de hacer (Darley, 2001, p. 207), entre el individuo como tal fuera de la organización y «otra persona» que entra en estado agentificado: en este caso nos referimos más bien a una serie de fuerzas sociales y organizacionales que impulsan y dirigen los comportamientos de las personas (nuevamente, no como autómatas sino justo como personas pensantes en una trama social siempre en movimiento).

Autoridad, obediencia, reglas y normas aceptadas, normalización y rutina, son todas fuerzas organizacionales que son capaces de impulsar complejas interpretaciones en las personas. La cuestión que sigue es, entonces, que las organizaciones son una fuerza social muy importante que intrínsecamente, de manera relativamente normal, producen ese tipo de estado agentificado, que al mismo tiempo que asegura obediencia y perseverancia para el logro de objetivos, puede, en ciertas circunstancias producir corrupción sistematizada y normalizada.

\section{Cooptación}

Uno de los instrumentos clásicos que las organizaciones usan para inducir cambios de comportamiento en las personas es a través de recordarles constantemente los beneficios adyacentes al salario de los que dichas personas gozan al pertenecer a las mismas (como el prestigio o la membresía a ciertos grupos). Ofrecer dichos beneficios como parte de la pertenencia a la organización ha sido conocido en la literatura como los «pagos colaterales» (Cyert y March, 1965). Si estos pagos colaterales devienen de actividades indebidas, pueden ser introducidos poco a poco como pagos que la acción y la cooperación otorgan aunque no estén formalizados. Incluso puede ser que la persona recién introducida no se dé cuenta de la posible malversación de recursos sino cuando ya ha pasado algún tiempo y ha recibido dichos pagos colaterales de manera constante. El punto central de la cooptación es justamente compartir intereses, ganancias y riesgos. Una vez introducida una persona a la red de relaciones y beneficios, es más difícil que dicha persona comprenda las implicaciones de su participación en las acciones y procesos organizativos, aun aquellos que pueden considerarse indebidos.

\section{Incrementalismo}

Diversos estudios sobre corrupción en organizaciones han notado que existe un proceso paulatino de introducción de las personas a la red de acciones y decisiones indebidas (Van Gennep, 1960; McLean y Elkind, 2004). El proceso puede iniciar con la encomienda a la persona recién llegada 
de pequeñas labores, a veces incluso insignificantes, pero que permiten a la misma introducirse a la cadena de diversas acciones que llevan a un producto o efecto organizacional. Esta clásica manera de introducir a una persona a la organización y sus procesos puede ser usada a su vez para efectos indebidos. De nueva cuenta, la persona puede que al principio no comprenda la problemática moral del efecto de su acción sino hasta que ya ha participado en la cadena cada vez con más responsabilidades y probablemente con una posición ahora para comprender mejor las consecuencias de sus actos. Es posible, además, que esta introducción incremental a la cadena de corrupción haga más difícil zafarse de la misma. La fuerza del pasado o de acciones ya concluidas entonces puede caer fuertemente sobre la persona, haciendo difícil que pueda tomar la decisión no solo de salirse sino incluso de denunciar (o como se conoce en la literatura, silbar para acusar whistleblowing).

\section{Compromiso}

La vida efectiva de una persona en una organización está llena de momentos donde debe enfrentar restricciones de muchos tipos, obligaciones normativas, presión para lograr objetivos con ciertos estándares y hacerlo en una vida organizacional de jerarquías, relaciones horizontales con otros, vínculos de grupos e incluso amistades o enemistades. No es extraño en estas circunstancias que alguno o algunos de estos elementos entren en contradicción. Lograr los objetivos con eficiencia puede implicar despedir gente de otros grupos o del propio. O tal vez acatar flexiblemente ciertas reglas o incluso desobedecerlas si por el bien del grupo o de los objetivos de la organización se trata. Por ejemplo, lograr resultados y estándares suele ser una de las presiones más fuertes en el mundo organizacional contemporáneo. La insistencia de que son los resultados lo único que importa, en contextos turbulentos e inciertos, impone a las personas una serie de presiones y angustias muy grandes ante la dificultad e incertidumbre de lograr dichos estándares o indicadores de resultados (véase los estudios experimentales de Lerner y Tetlock, 1999). De esta manera, un individuo puede llegar a aceptar flexibilidades en relaciones, reglas o procesos con el fin de lograr ciertos resultados o evadir ciertas consecuencias negativas. El compromiso con la organización, con el grupo, con los objetivos últimos, pueden ser fuerzas que hagan a las personas comenzar a flexibilizar sus patrones de acatamiento de normas o reglas, primero reducidamente, pero luego incrementalmente con flexibilidades cada vez mayores.

Como puede entenderse rápidamente, las dinámicas de racionalización y socialización se refuerzan mutuamente. Cuando se deja de lado el supuesto útil pero demasiado simple del decisor individual calculador y se comprenden tanto los mecanismos mentales y sicológicos para racionalizar y dar sentido a los actos propios, y se introducen en una arena de relaciones, reglas y tiempo organizacionales, la corrupción comienza a ser vista con otra perspectiva mucho más dinámica. Y también más preocupante pues puede intuirse la dificultad que tienen los mecanismos que han buscado lidiar con ella ${ }^{4}$.

\section{Conclusiones}

El objetivo de este artículo ha sido, a partir de una revisión de la literatura sobre corrupción organizacional, comprender el proceso de normalización de la corrupción. Este proceso es uno contra intuitivo y que puede frustrar a mucha gente que esperaría encontrar fórmulas rápidas

\footnotetext{
${ }^{4}$ Incluso hacerlo más allá de los nudges que no es claro generen un cambio cognitivo (Mols et al., 2014).
} 
y precisas para reducir el fenómeno (incluso algunos sueñan con eliminarlo completamente). La visión organizacional de la corrupción deja claro que no hay dicha «bala de plata» y que la corrupción es un fenómeno social de relaciones densas, donde lo «normal» y lo« anormal» se negocian e intersectan en las arenas donde los agentes terminan interactuando en el día a día.

Una sociedad y sus organizaciones pueden avanzar en reducir y acotar la corrupción. Y requieren de un proceso social interesante: analizar profundamente las prácticas, rutinas y racionalizaciones que definen sus relaciones como normales. Comprender, en otras palabras, cómo la lógica «normal» en que las personas se relacionan en una organización puede estar generando justamente el mismo fenómeno de la corrupción.

Desnormalizar la corrupción es, entonces, un paso necesario y sumamente difícil de realizar. Difícil porque implica adentrarse en las relaciones sociales, en los procesos que han convertido en rutina una serie de comportamientos que incluso pueden encontrarse ya racionalizados.

Se puede comenzar esta labor de comprender la desnormalización si se acepta que la corrupción es al final de cuentas una categoría social cargada enormemente de valores y expectativas (RoseAckerman, 2014, p. 4). No es el objetivo de esta conclusión discutir o posicionarse, por ejemplo, respecto de un argumento que ha sido atacado recientemente (incluso como extravagante, Caiden et al., 2001, p. 31): el que la corrupción puede ser un fenómeno inevitable y en algún sentido hasta benéfico socialmente en el largo plazo.

Dicha esta aclaración, es claro que para muchas personas se ha convertido en fundamental actuar respecto de la corrupción dados sus efectos negativos en muchos países y contextos. Los casos de corrupción generalizada en gobiernos como el mexicano, brasileño, guatemalteco (por mencionar algunos que en esta década han llamado la atención pública) y en empresas como Enron, Petrobras y Parmalat han llamado profundamente la atención social como casos profundamente preocupantes y graves. Todo esto empuja sin duda a diversos analistas a forzarse para proponer soluciones, de preferencia soluciones rápidas y universales. Se puede hablar incluso ya de una comunidad anticorrupción que ha adquirido su propia dinámica y lógica política. Sin embargo, como hemos propuesto aquí, la esperanza de que la corrupción sea eliminada de las relaciones sociales no es sencilla de satisfacer. Incluso imposible, si seguimos al extremo el argumento que acá hemos defendido. La visión organizacional de la corrupción resulta ser una fuente importante para comprender por qué. El agente sigue siendo el que cae en una lógica corrupta. Pero la decisión individual se da muchas veces en marcos sociales como las organizaciones (tal como hemos discutido aquí), y a la lógica individual de cálculo se le añade entonces la emocional o motivacional y la social o de las relaciones (Chugh et al., 2005, p. 78). Cada una de estas lógicas tiene su propia dinámica y los instrumentos anticorrupción que se piensen e implementen se verán afectados de diferente manera por ellas. Probablemente es por esto que la «batalla» contra la corrupción sea tan difícil y variable en resultados.

En todo caso, los actos corruptos son muchas veces un punto de llegada más que una decisión discreta de un actor individual autocontrolado: la interacción social de personas con emociones crea una dinámica, un lenguaje, un timing de interacción y actuación, y una racionalización que le permite al actor justificar su accionar. El espacio social decanta, prepara, normaliza la acción y las decisiones. Más importante, normaliza la interpretación para dar paso a una serie de acciones que se hacen casi automáticas, haciendo que ciertos actos sean normales. Normales porque otros lo hacen, normales porque otros los justifican. Comportamientos que se convierten en normales porque en su conjunto dado, la responsabilidad se ha repartido, atomizado, organizado. Pero tal vez lo más importante, porque al normalizar, el contexto organizacional y social desmitifica la vida social como algo alejado de los cuentos de hadas, con sus buenos vs. malos, con sus paladines 
vs. monstruos. Los grises son mucho más interesantes que la metáfora blanco y negro de la «lucha de los limpios contra los corruptos» ${ }^{5}$.

Desnormalizar la corrupción entonces parte de actuar sobre los procesos y rutinas que en la práctica ya están instalados en una organización, deconstruyéndolos de alguna forma, con el fin de comprender las cadenas casuales sociales y argumentativas que las sostienen. Comprender la corrupción como una interacción social posibilita así estudiar las reglas de acción, los elementos grupales y sicológicos que incitan y procrean comportamientos que terminan normalizando comportamientos que derivan en corrupción.

Regresando entonces al qué hacer, se requiere avanzar en los 3 frentes de desnormalización (a condición de no perder de vista la artificialidad): ¿qué cualidades individuales llevan a la corrupción?, ¿qué procesos organizacionales se generan, para normalizar los actos corruptos? y ¿cómo se refuerzan los procesos, reglas y normas organizacionales e institucionales para posicionar a los individuos a corruptos en esas posiciones o a los individuos a corromperse? Cuando la corrupción se normaliza, se institucionaliza, se hace sistémica en una organización o en un grupo de organizaciones, estas 3 dimensiones estarán presentes, recreándose.

\section{Referencias}

Ackroyd, S. y Thompson, P. (1999). Organizational misbehaviour. Londres: Sage. http://dx.doi.org/ $10.4135 / 9781446222232$

Ainslie, G. (2001). Break-down of will. Cambridge: Cambrdige University Press.

Anand, V., Ashforth, B., Joshi, M. y Martini, P. (2004). Business as usual: The acceptance and perpetuation of corruption in organizations. Academy of Management Executive, 18(2), 39-55.

Andersson, S. y Heywood, P. (2009). The politics of perception: Use and abuse of transparency international's approach to measuring corruption. Political Studies, 57, 746-767. http://dx.doi.org/10.1111/j.1467-9248.2008.00758.x

Arellano, D. (2010). Reformas administrativas y cambio organizacional: hacia el «efecto neto». Revista Mexicana de Sociología., 2010(2), 225-254.

Arellano, D. (2012). ¿Podemos reducir la corrupción en México? Límites y posibilidades de los instrumentos a nuestro alcance. México: CIDE.

Ashforth, B. y Anand, V. (2003). The normalization of corruption in organizations. Research in Organizational Behavior, 25, 1-52. http://dx.doi.org/10.1016/S0191-3085(03)25001-2

\footnotetext{
${ }^{5}$ Uno de los más graves obstáculos para comprender la corrupción probablemente tiene que ver, justamente, con la fuerza de los discursos moralistas y normativos que este concepto despierta en las sociedades contemporáneas. El concepto de corrupción contemporáneo forma parte de una forma dominante de comprender la sociedad moderna. Partiendo de una tajante y clara separación de lo público y lo privado, los conceptos más aceptados de corrupción tienen justamente que ver con ese ideal de separación de 2 esferas de intereses. Cuando el interés privado (legítimo en sí mismo) afecta negativamente el interés público (igualmente legítimo, este mucho más abstracto y cercano a un tipo ideal) es entonces cuando aparece la maleficencia de la corrupción. Lo público, puro, es adulterado por la intromisión inadecuada (se asumiría que hay una posible intromisión adecuada) de lo privado. Esta visión que sería extraña en la «premodernidad» implica siempre por tanto una definición normativa de lo que hace «puro» a lo «público» y de lo que hace dicha intromisión de lo privado, una intromisión inadecuada. Sin el mito o doctrina de «Los dos cuerpos del rey» (Kantorowicz, 1957), esos 2 cuerpos de todo ser humano, el concreto y el abstracto, el privado y el público, el concepto de corrupción contemporáneo es imposible de comprender. Parte del problema está, entonces, en no estar atentos a las contradicciones y limitaciones de una visión un tanto maniquea de los seres humanos en este marco normativo, socialmente creado en realidad, de la separación entre lo público y lo privado. Esa idea normativa, abstracta, construida social e históricamente (la de la separación de lo público y lo privado) es una conceptualización política a su vez, que busca imponer y normalizar ciertos comportamientos e ideas desde un marco hegemónico que intenta establecer lo que es «normal» (Bratsis, 2003, p. 17). Pero es justamente la ilusión de la pureza de lo público y la ilusión del autocontrol de lo privado para respetar dicha pureza lo que probablemente hace perder de vista precisamente cómo la corrupción o los comportamientos reales de las personas lidiando con la artificialidad de las esferas de lo público y lo privado son en sí mismos comportamientos en contextos, situaciones y lógicas sociales.
} 
Ashforth, B., Gioia, D., Robinson, S. y Treviño, L. (2008). Re-viewing organizational corruption. Academy of Management Review., 33(3), 670-684.

Bandura, A. (1990). Mechanisms of moral disengagement. En W. Reich (Ed.), Origins of terrorism: Psychologies, ideologies, theologies, states of mind (pp. 161-191). Cambridge: Cambridge University Press. http://dx.doi.org/10.5465/AMR.2008.32465714

Bargh, J. y Chartrand, T. (1999). The unbearable automaticity of being. American Psychologist, 54(7.), 462-479.

Benson, M. (1985). Denying the guilty mind: Accounting for involvement in a white-collar crime. Criminology., 23, 583-607. http://dx.doi.org/10.1111/j.1745-9125.1985.tb00365.x

Berger, P. y Luckman, T. (1978). La construcción social de la realidad. Buenos Aires: Amorrortu.

Bratsis'F P. (2003). The construction of corruption, or rules of separation and illusions of purity in Burgeois Societies. Social Text., 71(21-4), 9.33. http://dx.doi.org/10.1215/01642472-21-4_77-9

Caiden, G., Dwivedi, O. y Jabbra, J. (2001). Where corruption lives. Bloomfield: Kumarian Press.

Chibnall, S. y Saunders, P. (1977). Worlds apart: Notes on the social reality of corruption. The British Journal of Sociology., 28(2), 138-154.

Chugh, D., Bazerman, M. y Banaji, M. (2005). Bounded ethicality as a psychological barrier to recognizing conflicts of interest. En D. Moore, D. Moore, et al. (Eds.), Conflict of interest (pp. 74-95). Cambridge: Cambridge University Press.

Cialdini, R. B., Pterova, P. K. y Goldstein, N. J. (2004). The hidden costs of organizational dishonesty. Sloan Management Review, 45, 67-73.

Conklin, J. (1977). Business crime in America. Englewood: Prentice-hall.

Costas, J. y Grey, C. (2014). Bringing secrecy into de the open: Towards a theorization of the social processes of organizational secrecy. Organization Studies, 35(10), 1423-1447. http://dx.doi.org/10.1177/0170840613515470

Cressey, D. (1986). Why managers commit fraud. Australian and New Zealand Journal of Criminology, 19, $195-209$.

Cyert, R. y March, J. (1965). Teoría de las decisiones económicas de la empresa. México: Herrero.

Darley, J. (2001). The dynamics of authority influences in organizations and the unintended action consequences. En J. M. Darley, D. M. Messick, y T. R. Tyler (Eds.), Social influences on ethical behavior in organizations (pp. 37-52). New Jersey: Erlbaum.

De Graaf, G. (2007). Causes of corruption: Towards a contextual theory of corruption. Public Administration Review., $31(1 / 2), 39-86$.

De Graaf, G., Wagenaar, P. y Hoenderboom, M. (2010). Constructing corruption. En de G. de Graaf, P. von Maravic y P. Wagener (Eds). The good cause. Theoretical perspectives on corruption (pp. 98-114). Opladen \& Famington Hills: Barbara Budrich Pub.

Eagleman, D. (2012). Incognito. The secrets lives of the brain. Nueva York: Vintage.

Felps, W., Mitchell, T. y Byington, E. (2006). How, when, and why bad apples spoil the barrel: Negative group members and dysfunctional groups. Research in Organizational Behavior, 27, $175-222$. http://dx.doi.org/10.1016/S0191-3085(06)27005-9

Festinger, L. (1957). A Theory of cognitive dissonance. Stanford: Stanford University Press.

Fleming, P. y Zyglidopoulos, S. (2009). Charting corporate corruption. Northampton: Edward Elgar Pub.

Geis, G. y Meier, R. (1979). The white-collar offender. En H. Touch (Ed.), Psychology of crime and criminal justice.. Prospect Heights: Waveland Press.

Giddens, A. (1984). The constitution of society. Berkeley: University of California Press.

Gigerenzer, G. (2002). Bounded rationality: The adaptive toolbox. Cambridge: MIT Press.

Goffman, E. (1981). Forms of talk. Philadelphia: University of Pennsylvania Press.

Goffman, E. (1983). La presentación de la persona en la vida cotidiana. Buenos Aires: Amorrortu.

Haller, D. y Shore, C. (2005). Corruption. Anthropological perspectives. Londres: Pluto Press.

Kantorowicz, E. t. (1957). The king's two bodies. Princeton: Princeton University Press.

Kish-Gephart, J., Harrison, D. y Treviño, L. K. (2010). Bad apples, bad cases, and bad barrels: Metaanalytic evidence about sources of unethical decisions at work. Journal of Applied Psychology, 95(1), 1-13. http://dx.doi.org/10.1037/a0017103

Kunda, G. (1992). Engineering culture. Filadelfia: Temple University.

Lehrer, J. (2009). How we decide. Boston: Mariner Books. http://dx.doi.org/10.1037/0033-2909.125.2.255

Lerner, J. y Tetlock, P. (1999). Accounting for the effects of accountability. Psychological Bulletin, 125(2), $255-275$.

Lifton, R. (1986). The Nazi doctors: Medical killing and the psychology of genocide. Nueva York: Basic.

McLean, B. y Elkind, P. (2004). The smartest guys in the room. Nueva York: Portfolio Trade.

Messick, D. y Bazerman, M. (1996). Ethical leadership and the psychology of decision making. Sloan Management Review., 37(2), 9-22. 
Milgram, S. (2005). Obedience to authority. Nueva York: Printer \& Martin [1974].

Mols, F., Haslam, A., Jetten, J. y Steffens, N. (2014). Why a nudge is not enough: A social identity critique of governance by stealth. European Journal of Political Research., 54(1), 81-98. http://dx.doi.org/10.1111/1475-6765.12073

Nye, J. (1967). Corruption and political development: A cost benefit analysis. American Political Science Review., 61(2), $417-427$.

Philp, M. (1997). Defining political corruption. Political Studies, 45(3), 436-462.

Pinto, J., Leana, C. y Pil, F. (2008). Corrupt organizations or organizations of corrupt individuals? Two types of organization-level corruption. Academy of Management Review, 33, 685-709. http://dx.doi.org/10.5465/AMR.2008.32465726

Rest, J. (1986). Moral development: Advances in research and theory. Nueva York: Praeger.

Rose-Ackerman, S. (1978). Corruption: A study in political economy. Nueva York: Academic Press.

Rose-Ackerman, S. (2014). Corruption and conflict of interests. En J-B. Auby, E. Breen, y T. Perroud (Eds.), Corruption and conflict of interest. A comparative law approach (pp. 3-11). Cheltenham: Edward Elgar.

Simmel, G. (1906). The sociology of secrets and of secrets societies. American Journal of Sociology., 11(4), $441-498$.

Simon, H. (1947). Administrative behavior. Chicago: Chicago University Press.

Simon, H., Thompson, V. y Smithburg, D. (2010[1950]). Public administration. Nueva York: Transaction Publishers.

Sissener, T. (WP 2001). Anthropological perspectives on corruption. Bergen: Chr. Michelsen Institute., 5.

Staub, E. (1989). The roots of evil: The origins of genocide and other group violence. Nueva York: Cambridge University Press.

Sykes, G. y Matza, D. (1957). Techniques of neutralization: A theory of delinquency. American Sociological Review, 22, 664-670.

Turse, N. (2013). Killing everything that moves: The real American war in Vietnam. Nueva York: Picador.

Van Gennep, A. (1960). The rites of passages. Chicago: Chicago University Press.

Weick, K. (2001). Making sense of the organization. Oxford: Blackwell.

Zerubavel, E. (2006). The elephant in the room. Silence and denial in everyday life. Oxford: Oxford University Press.

Zyglidopoulos, S. y Fleming, P. (2008). Ethical distance in corrupt firms: How do innocent bystanders become guilty perpetrators? Journal of Business Ethics., 78(172.), 265-274. http://dx.doi.org/10.1007/s10551-007-9378-4

Zyglidopoulos, S., Fleming, P. y Rothenberg, S. (2009). Rationalization, overcompensation and the escalation of corruption in organizations. Journal of Business Ethics., 84(1), 65-73. http://dx.doi.org/10.1007/s10551-008-9685-4 